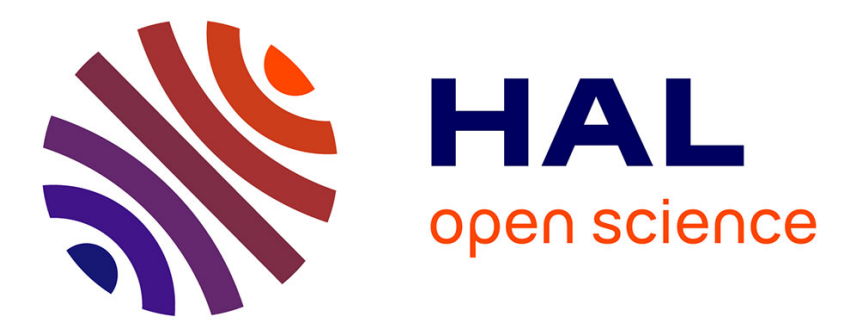

\title{
Données complémentaires sur les durées de mastication chez des vaches laitières recevant des rations riches en fourrage: influence de la nature du fourrage et du niveau d'apport d'aliment concentré
}

\author{
J.P. Dulphy, J. Rouel, M. Jailler, Daniel Sauvant
}

\section{To cite this version:}

J.P. Dulphy, J. Rouel, M. Jailler, Daniel Sauvant. Données complémentaires sur les durées de mastication chez des vaches laitières recevant des rations riches en fourrage: influence de la nature du fourrage et du niveau d'apport d'aliment concentré. Productions Animales, 1993, 6 (4), pp.297-302. hal-00896063

\section{HAL Id: hal-00896063 https://hal.science/hal-00896063}

Submitted on 1 Jan 1993

HAL is a multi-disciplinary open access archive for the deposit and dissemination of scientific research documents, whether they are published or not. The documents may come from teaching and research institutions in France or abroad, or from public or private research centers.
L'archive ouverte pluridisciplinaire HAL, est destinée au dépôt et à la diffusion de documents scientifiques de niveau recherche, publiés ou non, émanant des établissements d'enseignement et de recherche français ou étrangers, des laboratoires publics ou privés. 
INRA Prod. Anim., 1993, 6 (4), 297 - 302.
J.P. DULPHY, J. ROUEL*, M. JAILLER, D. SAUVANT**

INRA Station de Recherches sur la Nutrition des Herbivores

Theix 63122 Saint-Genès Champanelle

* INRA Domaine d'Orcival

63210 Rochefort-Montagne

** INRA-INA-PG

Station de Nutrition et d'Alimentation 16 rue Claude Bernard

75231 Paris Cedex 05
Données complémentaires sur les durées de mastication chez des vaches laitières recevant des rations riches en fourrage : influence de la nature du fourrage et du niveau d'apport d'aliment concentré

Les résultats présentés dans cet article complètent les données présentées par Sauvant et al en 1990 et la réflexion entreprise pour la mise en place d'un système de prévision de l'indice de fibrosité des fourrages.

Il existe, pour chaque aliment distribué à un ruminant, une durée totale de mastication par kg de MS (ingestion + rumination) nécessaire pour que celui-ci soit réduit en particules suffisamment fines pour quitter le rumen. La vitesse de transit des fines particules au niveau de l'orifice réticulo-omasal, ainsi que la vitesse de digestion des éléments digestibles de la ration sont alors les deux facteurs principaux qui déterminent le niveau d'ingestion des rations composées en majorité de fourrages.

Il est probable que la vache laitière, comme les autres ruminants, ne peut pas mastiquer au-delà d'une certaine durée journalière, cette

\section{Résumé}

La durée de mastication des rations est une donnée importante à prendre en compte lorsqu'on rationne des vaches laitières. C'est pourquoi les résultats obtenus pour 26 rations ont été récapitulés et ajoutés à 14 autre déjà publiés.

Pour les 40 rations concernées les animaux ont ingéré $11,4 \mathrm{~kg}$ de MS de fourrage et 5,8 kg d'aliment riche en énergie (proportion égale à $34 \%$ ). Les effets sur l'indice de fibrosité (durée de mastication par $\mathrm{kg}$ de MS ingérée) de la nature du fourrage, du niveau d'apport de concentré et du hachage dans une ration complète ont été précisés.

Les résultats ont été comparés à ceux de la littérature. La prévision des indices de fibrosité à partir de la teneur en cellulose brute des fourrages est discutée. Le recours à ce critère nécessite de distinguer les différentes catégories de fourrage (foin de graminées, foin de luzerne, ensilage de maïs, ensilage d'herbe).

Enfin les conditions nécessaires pour mettre en place un système précis de prévision de ces indices sont données. durée étant élevée puisque la valeur maximale de mastication citée par Jarrige (1978) est de $82,2 \%$ de la journée, soit $1184 \mathrm{~min} / \mathrm{j}$. A l'inverse les aliments concentrés sont mastiqués très rapidement et doivent être distribués avec des fourrages (Journet 1988). Les fourrages nécessitent un travail masticatoire important ; leur ingestion est donc limitée. Par contre, les fourrages et les rations qui nécessitent peu de mastication par kg de MS sont très ingestibles, mais leur ingestion risque d'entraîner des troubles digestifs car ils ne permettent pas une salivation suffisante. D'après Journet (1988), reprenant les conclusions de Sudweeks et al (1981) et celles de Kristensen et Norgaard (1987), une des composantes de la valeur hygiénique des rations peut être mesurée par le temps de mastication, qui dépend à la fois de la teneur en éléments fibreux (parois végétales) et de la structure physique des aliments (taille des particules).

La durée de mastication par kg de MS varie donc nécessairement dans des limites déterminées. Beaucoup de mesures ont déjà été faites et publiées. Ainsi Sauvant et al (1990) ont présenté les résultats relatifs à 50 rations. Pour compléter leur travail, nous avons enregistré, dans les mêmes conditions, les activités alimentaires et méryciques de vaches laitières pour 18 rations supplémentaires. Notre présentation concernera cependant l'ensemble des 40 rations que nous avons étudiées, les résultats de 14 d'entre elles ayant déjà été incluses dans l'étude de Sauvant et al (1990), et ceux de 8 autres l'étant dans celle de Dulphy et al (1990a). 


\section{1 / Matériel et méthodes}

Les animaux utilisés ont toujours été des vaches laitières de race Holstein, en lactation, conduites à l'INRA de Theix ou sur le Domaine d'Orcival. Pour chaque ration étudiée six animaux étaient choisis, en général dans des essais effectués pour d'autres buts. Une fois ces animaux bien adaptés à leur régime, quantités de MS ingérées et activités alimentaires étaient mesurées pendant 4 à 5 jours. A Theix les enregistrements d'activités alimentaires ont été effectuées selon la méthode de Ruckebusch (1963) et à Orcival selon la méthode de Brun et al (1984). Les animaux ont été caractérisés par leur poids vif et leur production de lait et les aliments par leur teneur en cellulose brute (méthode de Weende).

Les mesures concernant certains foins et les ensilages de maïs ont été faites avec des animaux spécialement conduits pour ces mesures. Pour les autres les animaux choisis étaient conduits dans le cadre des essais publiés par Dulphy et al (1990b), Dulphy et Rouel (1991) et Dulphy et al (1993).

Au total nous disposons donc de mesures pour 40 rations, qui correspondent à la distribution de 20 fourrages différents.

\section{2 / Résultats}

Les résultats détaillés concernant les 14 premières rations ont été donnés par Sauvant et al (1990). Ceux concernant les 26 autres sont donnés dans le tableau 1.

En moyenne, pour nos 40 rations, les animaux ont ingéré $11,4 \mathrm{~kg}$ de MS de fourrage et $5,8 \mathrm{~kg}$ de MS d'aliments riches en énergie (concentrés, betteraves dans 7 rations). Ces animaux pesaient $607 \pm 35 \mathrm{~kg}$ et produisaient $21,3 \pm 6,8 \mathrm{~kg}$ de lait à $4 \%$ de taux butyreux. La durée totale de mastication a été de $894 \pm 93 \mathrm{~min} /$ jour ( $62 \%$ du temps journalier) avec un minimum de 622 min et un maximum de $1105 \mathrm{~min}$. L'indice de fibrosité, c'est-à-dire la durée de mastication rapportée à la quantité ingérée, a été de $53,7 \pm 10,0 \mathrm{~min} / \mathrm{kg}$ de MS pour une teneur en cellulose brute de $20,7 \pm 3,8 \%$. Le pourcentage de concentré dans la ration a été de $27 \%$, ou $34 \pm 13 \%$ si on y rajoute les betteraves.

\section{1 / Effet de la nature du fourrage}

Pour estimer l'effet de la nature du fourrage nous avons fait l'hypothèse que la durée de mastication par $\mathrm{kg}$ de MS d'aliment concentré était de 10 min (cf. Sudweeks et al 1979) et celle des betteraves de 27 min (d'après nos calculs). L'étude de Sauvant et al (1990) attribuait respectivement $32,7 \mathrm{~min} / \mathrm{kg}$ au concentré et $45,3 \mathrm{~min} / \mathrm{kg}$ pour les betteraves. Nous n'avons pas retenu ces valeurs ici car elles intègrent des effets d'interaction sur le temps de mastication des fourrages. Les résultats moyens concernant les durées moyennes de mastication par $\mathrm{kg}$ de MS de ration et de fourrage figurent dans le tableau 2 .

\section{2 / Effet du niveau d'apport de concentré}

L'effet du niveau d'apport de concentré a été évalué à partir de 10 comparaisons entre un apport bas de concentré $(3,1 \mathrm{~kg}$ de $\mathrm{MS} /$ jour $)$ et un apport haut $(6,3 \mathrm{~kg}$ de $\mathrm{MS} /$ jour, betteraves comprises). L'augmentation de l'apport du concentré a fait baisser de $15 \%$ l'indice de fibrosité des rations, mais n'a pratiquement pas affecté celui des fourrages $(+3,5 \%)$ toujours sous l'hypothèse de $10 \mathrm{~min}$ de mastication / kg MS de concentré. Dans ces essais, l'ingestion de fourrage est passée de 11,9 à $10,2 \mathrm{~kg}$ de $\mathrm{MS} / \mathrm{j}$.

\section{3 / Effet de la distribution des aliments sous forme de ration complète}

Il y a eu deux comparaisons entre une ration dont le fourrage et le concentré étaient distribués séparément et une ration complète mélangée et hachée. Avec la ration complète l'indice de fibrosité a baissé de $14 \%$, soit $19 \%$ pour la partie fourrage. Dans le même temps la quantité totale ingérée est passée de 18,0 à $20,2 \mathrm{~kg}$ de $\mathrm{MS} /$ jour.

\section{4 / Variations de la durée journalière de mastication}

Une régression linéaire a été calculée entre la durée journalière de mastication d'une part et la quantité totale ingérée et la proportion de concentré d'autre part.

Dans les essais présentés ni la quantité totale de MS ingérée $(17,2 \pm 2,6 \mathrm{~kg})$, ni la proportion de concentré ( $34 \%$ ) n'ont eu d'effet significatif sur la durée journalière de mastication des rations $(\mathrm{R}=0,11)$, mais l'écart-type résiduel a été élevé ( $\pm 106 \mathrm{~min}$ ), et donc à l'origine du manque de signification.

\section{5 / Variations de l'indice de fibrosité des rations}

Il existe des relations relativement étroites entre l'indice de fibrosité des rations, la quantité totale ingérée, la proportion de concentré, ainsi que la teneur en cellulose brute. Ces relations (régressions linéaires multiples) sont données dans le tableau 3.

\section{3 / Discussion}

Les données présentées complètent celles fournies par Sauvant et al (1990). Elles correspondent en général à des rations contenant moins d'aliments concentrés en proportion, et dont les quantités ingérées de MS sont un peu plus faibles. Ces rations sont donc, logiquement, mastiquées plus longtemps.

Si nous appliquons aux données récapitulées par Sauvant et al (1990) l'hypothèse que nous avons appliquée pour nos données, il est possible d'estimer les indices de fibrosité de fourrages supplémentaires. Pour 7 ensilages 
Tableau 1. Caractéristiques d'ingestion et de mastication des 26 rations complémentaires.

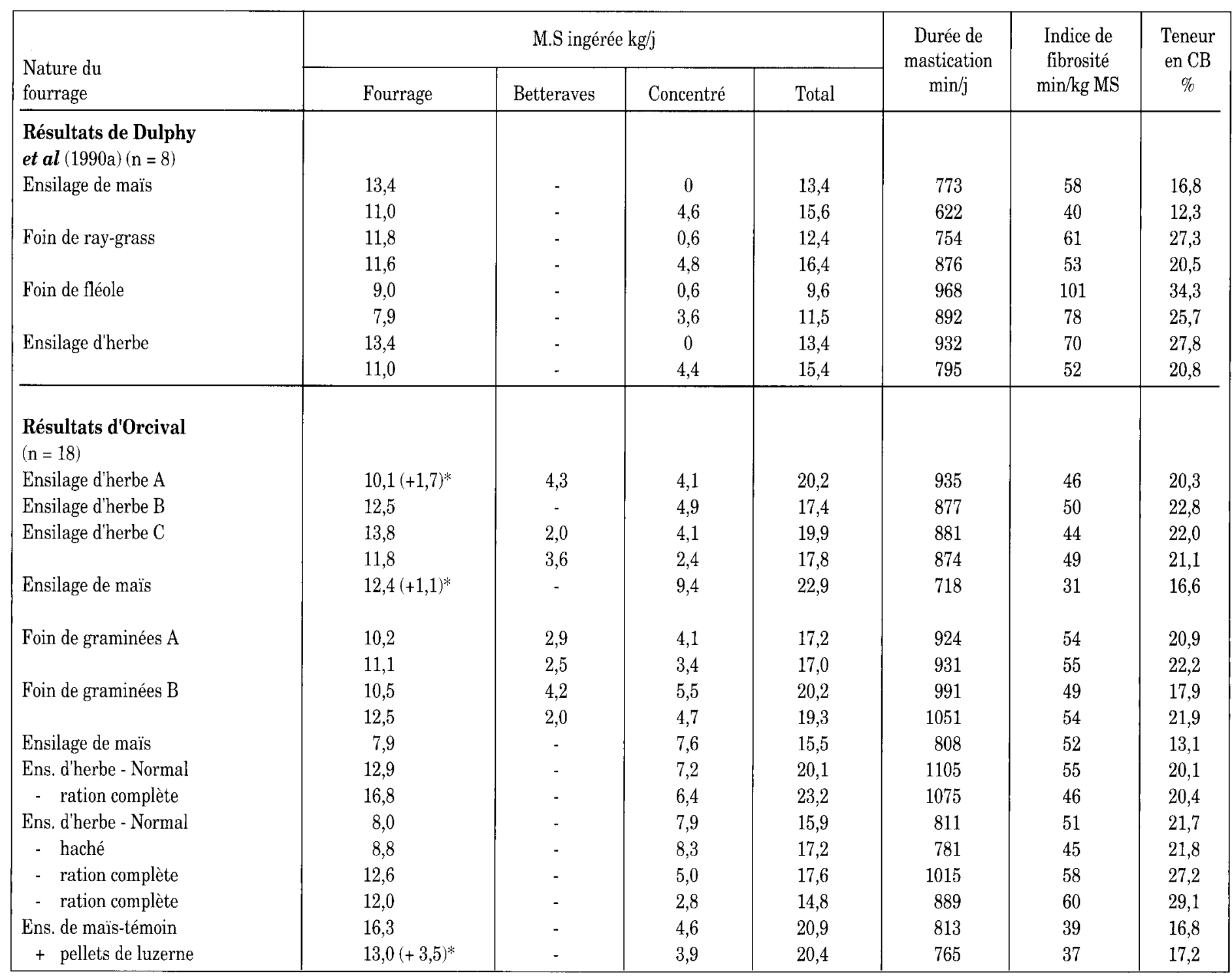

$*$ (foin)

Tableau 2. Durée de mastication des différentes rations.

\begin{tabular}{|c|c|c|c|c|}
\hline & \multicolumn{2}{|c|}{$\begin{array}{l}\text { Durée de mastication par kg de MS } \\
\qquad(=\text { indice de fibrosité })\end{array}$} & \multicolumn{2}{|c|}{ Teneur en CB \% } \\
\hline & Ration & Fourrage & Ration & Fourrage \\
\hline $\begin{array}{l}\text { Nature du fourrage } \\
\text { - Ensilage de maïs } \\
\text { - Foin } \\
\text { - Ensilage d'herbe }\end{array}$ & $\begin{array}{c}46,8 \pm 8,3^{\mathrm{b}}(12) \\
62,9 \pm 16,2^{\mathrm{a}}(10) \\
53,2 \pm 9,6^{\mathrm{b}}(18)\end{array}$ & $\begin{array}{c}62,0 \pm 20,2^{\mathrm{b}(5)} \\
82,0 \pm 16,2^{\mathrm{a}}(6) \\
72,6 \pm 12,4^{\mathrm{ab}(9)}\end{array}$ & $\begin{array}{l}16,1 \\
23,9 \\
22,8\end{array}$ & $\begin{array}{l}18,4 \\
29,9 \\
28,7\end{array}$ \\
\hline $\begin{array}{l}\text { Apport de concentré } \\
\text { - Bas } \\
\text { - Haut }\end{array}$ & $\begin{array}{l}63,6 \pm 14,7 \mathrm{a}(10) \\
54,1 \pm 10,1^{\mathrm{a}}(10)\end{array}$ & $\begin{array}{l}75,0 \pm 13,4 \mathrm{a}(9) \\
77,6 \pm 14,3 \mathrm{a}(9)\end{array}$ & $\begin{array}{l}24,1 \\
20,0\end{array}$ & 26,8 \\
\hline $\begin{array}{l}\text { Nature de la ration } \\
\text { - Séparée } \\
\text { - Complète }\end{array}$ & $\begin{array}{l}53,0(2) \\
45,5(2)\end{array}$ & $\begin{array}{l}85,5(2) \\
69,5(2)\end{array}$ & $\begin{array}{l}20,9 \\
21,1\end{array}$ & 28,6 \\
\hline
\end{tabular}

() : nombre de données

A lintérieur de chaque case, les résultats suivis par une lettre différente sont significativement différents au seuil de $5 \%$. 
Tableau 3. Valeurs des paramètres des régressions multiples entre indices de fibrosité d'une part, quantités totales ingérées, proportion de concentré et teneur en cellulose brute d'autre part.

\begin{tabular}{|c|c|c|c|c|c|c|}
\hline \multicolumn{2}{|c|}{ Equation $n^{\circ}$} & 1 & 2 & 3 & 4 & 5 \\
\hline \multicolumn{2}{|c|}{ Constante } & 112 & 189 & 113 & 11,3 & 73 \\
\hline Pente & QIT & $\begin{array}{l}-3,15 \\
(0,47)\end{array}$ & $\begin{array}{c}-12,84 \\
(3,4)\end{array}$ & $\begin{array}{c}-3,43 \\
(0,4) \\
\end{array}$ & - & $\begin{array}{l}-2,53 \\
(0,37)\end{array}$ \\
\hline des & $\mathrm{QIT}^{2}$ & - & $\begin{array}{c}0,279 \\
(0,097) \\
\end{array}$ & - & - & - \\
\hline \multirow{2}{*}{ variables } & $\mathrm{PC}$ & $\begin{array}{c}-0,117 \\
(0,105) \\
\end{array}$ & $\begin{array}{c}0,007 \\
(0,105)\end{array}$ & - & - & . \\
\hline & $\mathrm{CB}$ & - & - & - & $\begin{array}{l}2,018 \\
(0,31) \\
\end{array}$ & $\begin{array}{c}1,16 \\
(0,25) \\
\end{array}$ \\
\hline \multicolumn{2}{|c|}{$\mathrm{R}$} & 0,82 & 0,86 & 0,81 & 0,72 & 0,89 \\
\hline \multicolumn{2}{|c|}{ ETR } & 7,3 & 6,7 & 7,4 & 8,7 & 5,9 \\
\hline
\end{tabular}

QIT : Quantités totales de MS ingérées ( $\mathrm{kg}$ )

$\mathrm{PC}:$ Proportion de concentré (\%)

CB : Teneur en cellulose brute (\%)

( ) : Ecart-type des paramètres

$R$ : Ceefficient de corrélation multiple

ETR : Ecart-type résiduel de la régression

de mais cet indice moyen est de $70 \pm 13 \mathrm{~min} / \mathrm{kg}$ MS et pour 3 foins de graminées ingérés en quantité notable il est de $84 \pm 20$. Il semble nettement plus faible pour les foins de luzerne $(47 \pm 13$ min pour $n=11)$. Il n'y a pas de données pour l'ensilage d'herbe.

Sudweeks et al (1981) fournissent aussi quelques données : 40 à $66 \mathrm{~min} / \mathrm{kg}$ de MS pour l'ensilage de maïs, 77 à 109 pour des foins de graminées, 99 à 120 pour des ensilages d'herbe. Cependant ces données ne peuvent être directement comparées aux nôtres, faute de critères communs caractérisant les fourrages.

Le système danois (Norgaard 1983) de prévision de l'indice de fibrosité (IF) pour les fourrages est très simple :

$\mathrm{IF}=2,25 \mathrm{CB}$ pour un hachage grossier (10-

$50 \mathrm{~mm}$ )

IF $=3 \mathrm{CB}$ pour des fourrages longs

Les ensilages de mais, dont la longueur moyenne des particules est de $5-10 \mathrm{~mm}$ dan's nos essais, s'éloignent nettement de ce modèle. Pour eux IF/CB $=3,37$, valeur encore plus élevée probablement dans les mesures récapitulées par Sauvant et al (1990). Il serait intéressant à l'avenir de préciser l'effet de la finesse de hachage du maïs et de confirmer la valeur relativement élevée de l'indice de fibrosité de ces fourrages par rapport aux fourrages classiques de graminées.

Dans nos observations les ensilages d'herbe ont un rapport IF/CB égal à 2,54 pour des longueurs moyennes de brins comprises entre 10 et $20 \mathrm{~mm}$. Cette valeur est proche de celle de Norgaard (1983), bien que très légèrement supérieure $(+13 \%)$.
Pour ce qui concerne les foins de graminées les 3 résultats considérés dans la base de Sauvant et al (1990) sont proches des nôtres. Pour ces derniers nous avons IF/CB égal à 2,74 alors que les Danois proposent 3,0.

Il n'y a malheureusement pas de foins de luzerne dans nos observations. D'après les résultats donnés par Sauvant et al (1990) l'indice de ces foins serait faible, en comparaison de leurs teneurs en cellulose brute élevées. Ils devraient donc avoir des valeurs IF/CB proches de 1,5 .

Pour 18 fourrages, Norgaard (1990) donne une relation entre indice de fibrosité et teneur en CB :

$$
\mathrm{IF}=-37,7+4,23 \mathrm{CB}
$$

Cette relation n'est pas du tout adaptée aux ensilages de maïs ; elle donne des résultats un peu supérieurs aux nôtres pour les foins $(+7 \%)$ et les ensilages d'herbe $(+15 \%)$.

L'étude comparée de différents fourrages confirme donc que la teneur en cellulose brute est peut-être un critère de prévision utilisable, mais uniquement à l'intérieur d'une catégorie de fourrage (foin de graminées, foin de luzerne, ensilage de maïs, ensilage d'herbe). Si on veut faire abstraction de la nature du fourrage, il faut choisir d'autres critères. Sauvant et al (1990) ont considéré le résidu NDF, car il est fréquemment cité dans la littérature récente. Cependant il reste un gros travail à faire pour le confirmer. En effet Kaiser et Combs (1989) montrent que ce critère est lui aussi insuffisant.

Pour calculer l'indice de fibrosité d'une ration on fait l'hypothèse que les indices du 
fourrage et du concentré sont constants. Il est probable que cela n'est pas tout à fait le cas. Dans nos essais on observe en effet une tendance, non significative, à l'augmentation de l'indice de fibrosité estimé du fourrage lorsque l'apport du concentré augmente. Guerin et Dulphy (1984) notent la même tendance chez des moutons. Le phénomène pourrait s'expliquer par une baisse de la vitesse de digestion du fourrage (Berge et Dulphy, 1985 et 1991) nécessitant une mastication plus poussée, cependant il faudrait pouvoir mesurer plus précisément cet effet.

Pour prévoir l'indice de fibrosité des fourrages un autre élément très important est leur finesse de hachage (Santini et al 1983). Les Danois en tiennent compte de façon très grossière et nos deux essais sur rations complètes confirment l'importance de ce paramètre qui reste à analyser plus précisément, non seulement pour les ensilages d'herbe, mais encore pour les ensilages de maïs dont la finesse de hachage est, en pratique, très variable.

$\mathrm{Au}$ niveau des rations les régressions multiples que nous avons calculées sont comparables à celles de Sauvant et al (1990). Pour des quantités ingérées totales de $18 \mathrm{~kg}$ de MS et une proportion de concentré de $30 \%$, l'équation (1) de ce travail et l'équation (5) de Sauvant et al (1990) fournissent les mêmes valeurs de $52 \mathrm{~min} / \mathrm{kg}$ de MS, ce qui montre qu'il existe une continuité entre les 2 populations de régimes étudiés. L'équation (2) de ce travail et l'équation (6) de Sauvant, qui incluent un terme quadratique donnent des résultats légèrement différents : respectivement 48 et $45 \mathrm{~min} / \mathrm{kg}$ MS pour $18 \mathrm{~kg}$ de MS totale ingérée et $30 \%$ de concentré dans la ration. Ces 4 équations, même si elles ont peu d'intérêt pour prévoir des indices de fibrosité, montrent que les quantités ingérées de fourrage et de concentré sont reliées aux indices de fibrosité et qu'il existe donc des possibilités d'utiliser directement des caractéristiques des aliments pour prévoir l'indice de fibrosité des rations. Ces relations sont cependant accompagnées d'un écart-type résiduel élevé, de l'ordre de $7 \mathrm{~min} / \mathrm{kg}$ de MS, au même titre d'ailleurs que celles incluant la teneur en cellulose brute. Cela confirme bien que les facteurs précisément responsables des variations de l'indice de fibrosité sont encore mal connus.

Cette discussion permet de comprendre que la mise en place d'un système précis de prévision de l'indice de fibrosité des fourrages ne peut être envisagée dans un avenir proche. Pour ce faire il faut en effet, comme le soulignent Sauvant et al (1990) définir un protocole standard de mesure, vérifier sa répétabilité, déterminer des lois de corrections pour tenir compte des caractéristiques des animaux, mesurer l'effet de la variation du niveau d'apport de l'aliment concentré, rechercher des caractéristiques qualitatives des fourrages à prendre en compte, plus généralisables que la teneur en cellulose brute.

Compte tenu du nombre de mesures répétitives à faire, un programme spécifique serait particulièrement lourd, d'autant plus qu'il devrait s'accompagner de mesures complémentaires sur les caractéristiques physico-chimiques des contenus ruminaux et sur l'état des animaux afin de pouvoir prendre en compte plus correctement les indices de fibrosité dans les conseils de rationnement. Il s'agit en effet, dans un premier temps, de comprendre comment éviter des problèmes pathologiques (Sudweeks et al 1981). Mais au-delà il est sûr que la maîtrise des caractéristiques physicochimiques des contenus ruminaux est indispensable pour obtenir une digestion optimale dans le rumen (cf. par exemple Beauchemin et Buchanan-Smith, 1989 et 1990). Cette maîtrise à travers la nature des aliments est une alternative intéressante aux substances tampons qui ont suscité tant de recherches (Erdman 1988).

Par ailleurs il sera nécessaire, mais difficile, de pratiquer des mesures et de définir des lois de prévision pour les concentrés et pour l'ensemble des sous-produits utilisés en alimentation compte tenu de leur grande diversité. De nombreuses mesures systématiques seront alors de toute façon nécessaires pour compléter les observations déjà publiées.

Enfin les travaux concernant l'influence des caractéristiques physiques des aliments de ruminants sur leur durée de mastication mériteront d'être poursuivis, en particulier ceux entrepris au Danemark (Norgaard 1986, $1987 \mathrm{a}, 1987 \mathrm{~b}, 1989 \mathrm{a}, 1989 \mathrm{~b}, 1989 \mathrm{c}$ ). Cependant, avec les résultats déjà connus, une prise en compte de cette influence par le biais des indices de fibrosité, même si elle est encore imprécise, est tout à fait possible.

Pour conclure, l'intérêt du présent travail est d'augmenter très nettement la base de données, commencée par Sauvant et al (1990), avec des rations relativement riches en fourrages. Il est aussi de compléter la réflexion entreprise pour la mise en place d'un système que réclament nombre de nos partenaires.

\section{Références bibliographiques}

Beauchemin K.A., Buchanan-Smith J.G., 1989. Effects of dietary neutral detergent fiber concentration and supplementary long hay on chewing activities and milk production of dairy cows. J. Dairy Sci., 72, 2288-2300.

Beauchemin K.A., Buchanan-Smith J.G., 1990. Effects of fiber source and method of feeding on chewing activities, digestive function, and productivity of dairy cows. J. Dairy Sci., 73, 749-762.

Berge P., Dulphy J.P., 1985. Etude des interactions entre fourrage et aliment concentré chez le mouton. I Facteurs de variation du taux de substitution. Ann. Zootech., 34, 313-334.

Berge P., Dulphy J.P., 1991. Etude des interactions entre fourrage et aliment concentré chez le mouton. II Facteurs de variation de la digestibilité. Ann. Zootech., 40, 227-246. 
Brun J.P., Prache S., Béchet G., 1984. A portable device for eating behavior studies. 5th Meeting Eur. Grazing Workshop

Dulphy J.P., Rouel J., 1991. Association de betteraves fourragères à du foin pour des vaches laitières. Ann. Zootech. 40, 201-207.

Dulphy J.P., Carle B., Demarquilly C., 1990a Quantités ingérées et activités alimentaires comparées des ovins, bovins, et caprins recevant des fourrages conservés avec ou sans aliment concentré. Ann. Zootech. 39, 95-111.

Dulphy J.P., Rouel J., Bony J., 1990b. Association de betteraves fourragères à de l'ensilage d'herbe pour des vaches laitières. INRA Prod. Anim., 3, 3, 195-200.

Dulphy J.P., Rouel J., Bony J., Andrieu J.P., 1993. Valeur alimentaire des rations complètes à base d'ensilage d'herbe pour les vaches laitières. Ann. Zootech., sous presse.

Erdman R.A., 1988. Dietary buffering requirements of the lactating dairy cow : a review. J. Dairy Sci., 71, 3246-3266.

Guerin H., Dulphy J.P., 1984. Influence de l'apport complémentaire de maîs, de pulpe de betterave ou de mélasse sur la valeur alimentaire d'un foin. Ann. Zootech., 33, 509-532.

Jarrige R. 1978. Consommation d'aliments et d'eau. in : "Alimentation des Ruminants". Ed. INRA Publications, Route de St-Cyr, 78000 Versailles. p. 177-206.

Journet M. 1988. Optimisation des rations. in $\mathrm{R}$. Jarrige ed. : "Alimentation des bovins, ovins, caprins". Ed. INRA Publications. 121-134.

Kaiser R.M., Combs D.K., 1989. Utilization of three maturities of Alfalfa by dairy cows fed rations that contain similar concentrations of fiber. J. Dairy Sci. 72, 2301-2307

Kristensen V. Friis, Norgaard P., 1987. Effect of roughage quality and physical structure of the diet on feed intake and milk yield of the dairy cow. Cattle Production Research. Danish Status and Perspectives. Landhusholdvningsselskabets Forlag, Copenhagen. p. 79-91

Norgaard P., 1983. Optimale foderrationel til malkekoen, fodervaerdi, foderoptagelse, omsaetning $0 \mathrm{~g}$ produktion. Rep. 551 Nat. Inst. Anim. Sci., Copenhagen.
Norgaard P., 1986. Physical structure of feeds for dairy cows in new developments and future perspectives in research on rumen function. Neimann-Sorensen A. Ed. Com. Europ. Com. Luxembourg, p. 85-107.

Norgaard P., 1987a. The influence of level of feeding and physical form of the feed on the reticulo-rumen fermentation in dairy cows fed 12 times daily. Acta Agric. Scand. 37, 353-365.

Norgaard P., 1987b. Reticulo-rumen fermentation in dairy cows fed 12 times daily with 4 types of roughages supplemented with straw and concentrates. Acta Agric. Scand. 37, 449-461.

Norgaard P., 1989a. The influence of physical form of ration on chewing activity and rumen motility in lactating cows. Acta Agric. Scand. 39, 187-202

Norgaard P., 1989b. Influence of physical form of the ration on ruminal ingesta and ruminal fermentation in lactating cows fed 12 times daily. Acta Agric. Scand. 39, 441-448.

Norgaard P., 1989c. Influence of the physical form of diet on chewing activity and reticulo-rumen motility in cows. Act. Vet. Scand. Suppl, $n^{\circ} 86,46-52$

Norgaard P., 1990. The use of time spent chewing in the formulation of optimal rations for dairy cows. Kolloquium, Kurzfassungen der Vorträge, 6 dezember, Leipzig.

Ruckebusch Y., 1963. Recherches sur la régulation centrale du comportement alimentaire chez les ruminants. Thèse Doct-ès-Sciences. Université de Lyon. 213 p.

Santini F.I., Hardie A.R., Jorgensen N.A., Finner M.F., 1983. Proposed use of adjusted intake based on forage particle length for calculation of roughage indexes. J. Dairy Sci., 66, 811-820.

Sauvant D., Dulphy J.P., Michalet-Doreau B., 1990. Le concept d'indice de fibrosité des aliments des ruminants. INRA Prod. Anim., 3, 309-318.

Sudweeks E.M., Anderson M.J., Lamb R.C., Law S.E., Ely L.O., Mc Cullough M.E., Sisk L.R., 1979 Development and application of a roughage value index for formulating dairy rations. Georgia Exp. Sta. Res. Bull. 238

Sudweeks E.M., Ely L.O., Mertens D.R., Sisk L.R., 1981. Assessing amounts and form of roughages in ruminants diets : roughage value index system. J. Anim. Sci. 53, 1406-1411.

\section{Summary}

Additional data on mastication time in dairy cows fed forage-rich diet : influence of the forage type and concentrate supplementation level.

Diet mastication time is a relevant datum that should be taken into account when preparing dairy cows' diets. For this reason, the results obtained with 26 diets were summarized and compiled with those already published on 14 diets.

For the 40 studied diets, animals ingested $11.4 \mathrm{~kg}$ forage dry matter and $5.8 \mathrm{~kg}$ energy-rich feed (proportion $=34 \%$ ). The effects on fibrosity (mastication time per $\mathrm{kg}$ DM ingested) of the type of forage, concentrate supplementation level and chopping of a complete diet, are documented.
Results were compared with those already published. Fibrosity index prediction from forage cellulose contents is discussed. Using that criterion requires distinguishing the different types of forage (grass hay, lucerne hay, maize silage, grass silage).

Lastly, the conditions required to set up an accurate prediction system for the diet fibrosity are described.

DULPHY J.P., ROUEL J., JAILLER M., SAUVANT D. 1993. Données complémentaires sur les durées de mastication chez des vaches laitières recevant des rations riches en fourrage : influence de la nature du fourrage et du niveau d'apport d'aliment concentré. INRA Prod. Anim., 6 (4), 297-302. 